\title{
The Nanomaterial Registry: facilitating the sharing and analysis of data in the diverse nanomaterial community
}

This article was published in the following Dove Press journal:

International Journal of Nanomedicine

12 September 2013

Number of times this article has been viewed

\section{Michele L Ostraat \\ Karmann C Mills \\ Kimberly A Guzan \\ Damaris Murry}

RTI International, Durham, NC, USA

Correspondence: Michele L Ostraat RTI International,

3040 East Cornwallis Road, Durham,

NC 27709, USA

Email mostraat@rti.org
Abstract: The amount of data being generated in the nanotechnology research space is significant, and the coordination, sharing, and downstream analysis of the data is complex and consistently deliberated. The complexities of the data are due in large part to the inherently complicated characteristics of nanomaterials. Also, testing protocols and assays used for nanomaterials are diverse and lacking standardization. The Nanomaterial Registry has been developed to address such challenges as the need for standard methods, data formatting, and controlled vocabularies for data sharing. The Registry is an authoritative, web-based tool whose purpose is to simplify the community's level of effort in assessing nanomaterial data from environmental and biological interaction studies. Because the Registry is meant to be an authoritative resource, all data-driven content is systematically archived and reviewed by subject-matter experts. To support and advance nanomaterial research, a set of minimal information about nanomaterials (MIAN) has been developed and is foundational to the Registry data model. The MIAN has been used to create evaluation and similarity criteria for nanomaterials that are curated into the Registry. The Registry is a publicly available resource that is being built through collaborations with many stakeholder groups in the nanotechnology community, including industry, regulatory, government, and academia. Features of the Registry website (http://www.nanomaterialregistry. org) currently include search, browse, side-by-side comparison of nanomaterials, compliance ratings based on the quality and quantity of data, and the ability to search for similar nanomaterials within the Registry. This paper is a modification and extension of a proceedings paper for the Institute of Electrical and Electronics Engineers.

Keywords: nanoinformatics, Registry, minimal information standards

\section{Introduction}

The growth of research and knowledge in nanotechnology presents the community with both challenges and opportunities. Not only are nanomaterial stakeholder groups diverse, but the amount of data and metadata being reported can be vast, complex, challenging to interpret, and vary in quality. There are also many gaps in the published data, and a lack of standardized protocols and measurement schemes. ${ }^{1-3}$ As a result, the need for accepted minimal information standards, controlled vocabularies, standard data formats, and appropriate and publicly available tools to access, visualize, and analyze nanomaterial data is well documented in the literature. ${ }^{4-7}$

Several opportunities to address the aforementioned challenges and to encourage cooperative research within the nanotechnology community have been identified, and a number of efforts as well as resources and tools are under development. 
For example, efforts to build consensus around a minimal information standard on nanomaterial characterization have been made, ${ }^{8-10}$ although to date none has been adapted community-wide. Efforts to establish standardized data formatting to support data interoperability are also under way. ${ }^{11}$ Other efforts include establishing controlled vocabularies $^{12}$ and building ontologies focused on nano/ bio-interactions. ${ }^{13}$

The Nanomaterial Registry has been established to provide an authoritative, web-based tool for data analysis. Existing data are curated into the Registry based on the minimal information about nanomaterials (MIAN) criteria, which was developed by the Registry team, and is specific to physicochemical characterization (PCC) and studies on biological and environmental interactions of nanomaterials. The MIAN has been used to establish evaluation and similarity measures for nanomaterial data. Community efforts in the areas listed above - particularly those of controlled vocabularies, data interoperability, and ontology - are being built upon to develop a tool that supports nanomaterial data assessment. This paper is a modification and extension of a proceedings paper for the Institute of Electrical and Electronics Engineers (IEEE). ${ }^{14}$

\section{Content of the Nanomaterial Registry \\ Nanomaterial Registry data model}

Two concepts that were foundational to the design of the Registry data model were the MIAN and the need for flexibility. The Registry data model is based on the MIAN, which was built through collaborations with and guidance from an advisory board made up of representatives from stakeholder groups in the nanotechnology community, including industry, regulatory, government, and academia. The work of minimal information working groups, such as the MINChar Initiative, was also influential to the MIAN's development. ${ }^{15}$ The MIAN identifies the PCC data and metadata that should be reported in order to facilitate interpretation, sharing, and downstream analysis of data that are generated from disparate sources on nanomaterial interactions with biological and environmental systems. The other driving concept in the development of the Registry data model was the need to have a tool that is flexible and expandable, so that new community developments, standards, or future discoveries can be captured, reflected, and used in the Registry. The data model was built in a way that allows for easy addition of data points and relational information as well as the flexibility to expand data classes and categories.
Several aspects make the Registry unique. Most web-based nanomaterial tools contain information that is subject area-specific. In contrast, the Registry's mission is to collect and share a broad body of nanomaterial knowledge. Therefore, the data model that has been designed and implemented enables the curation of data that is necessarily broad rather than detailed and that spans, for example, environmental fate and transport, occupational health and safety, and biotoxicology. Potential data sources for inclusion in the Registry are vetted through a process that includes the following criteria:

- How well does the scope of their data match the scope of the Registry?

- What type of database is the source? (ie, curated data, papers, or paper abstracts)

- Were the studies peer-reviewed?

- Have the raw data from the study been published and/or could the data be made publicly available?

- How many PCCs in the Registry's MIAN does the data source provide for each nanomaterial?

- Are there data available from biological and environmental interaction studies?

- Are measurement protocols and techniques usually reported?

- Are the data in the data source separated into individual fields, or are they in paragraphs of free text?

- What were the original sources of the data? (ie, papers or individual investigators).

Once vetted, the data from the source would be curated and made available to the public on the Registry site. Currently, the data being integrated into the Registry comes from publicly available sources. In order to have data included in the Registry, one approach would be to submit your data to one of the data sources currently being used to populate the Registry. The vision for the future of the Registry includes the production and release of a data-entry tool for the public. Data submitted via the data-entry tool could come from individual researchers or manufacturers, and would be curated in the same way as data from large data sources.

In addition to housing multidisciplinary data, the Registry data model incorporates the concept of instance of characterization (IOC; discussed below) in order to capture changes in a nanomaterial that are due to changes in its environment, and ultimately in order to aid in the validation of data comparison. This concept is key to the data model and the web-based data display, because a single nanomaterial may have multiple sets of data, each corresponding to a different IOC. 
Finally, a controlled vocabulary was established for the Registry MIAN by leveraging external efforts, and descriptors for each term were outlined in regard to how the Registry would use each term (available in the online glossary). Having a controlled vocabulary ensured that all data curated to the Registry would be easily comparable. For example, in order to avoid curating many unique names for shapes based on how the data source describes the nanomaterial, the Registry used dimensionality in the nanoscale to designate shape based upon the definitions of an International Organization for Standardization (ISO) vocabulary standard (1-D, 2-D, 3-D). ${ }^{16,17}$

\section{Minimal information about nanomaterials}

The scope of the Registry PCC MIAN, listed in Table 1, includes the characteristics of a nanomaterial that govern its interactions in biological and environmental systems. The Registry's PCC MIAN captures not only characteristic data but also the protocols and parameters associated with each measurement of those characteristics and general information about the nanomaterial, such as production or synthesis technique, product name, lot number, and manufacturer. All of this information is viewable on the Registry website from the nanomaterial details page.

For each PCC, there are three different subsets of information required in the minimal information standards: (1) measurement values; (2) analysis technique, instrument, and protocol and parameter information; and (3) scientific method and best-practice evaluation. The MIAN was designed this way because there are many instances in the literature in which instrument parameters and experimental protocols can greatly impact analysis. The goal for this level of comprehensiveness is to encourage the adoption of greater reporting in literature and promote standards creation about data collection and communication.

The Registry has also recently completed a minimal information standard to be used for the curation of studies performed on the biological and environmental implications of nanomaterials. The minimal information for studies ensures that the data from studies are curated in a way that summarizes the vital information in the study and helps the user better interpret how the study was performed and the outcomes. The four primary categories curated for each study are:

- General study details - includes test location, study controls, assay and standard protocol information

- Test subject/media - includes specifics about in vivo, in vitro, or air/soil/water test types

- Exposure summary - includes method of exposure, dosage, frequency, and duration information

- End points - includes toxicity, mechanistic cellular uptake, biotransformation, and fate and transport information.

The definition of the Registry MIAN is expected to evolve as research and technology progress, and as the body of knowledge about nanomaterials expands. Overtime, the

Table I Descriptors of the Nanomaterial Registry minimal information about nanomaterials (MIAN)'s physicochemical characterization (PCC)

\begin{tabular}{ll}
\hline PCC & Descriptor used in the Nanomaterial Registry \\
\hline $\begin{array}{l}\text { Composition } \\
\text { Size* }\end{array}$ & $\begin{array}{l}\text { Chemical information and crystal structure of the nanomaterial } \\
\text { The physical dimensions of a particle. For spherical particles, it is their diameter. For irregularly shaped particles, it is the } \\
\text { diameter of the equivalent sphere that has the same volume as a particle. Also includes physical state of the sample }\end{array}$ \\
Size distribution & $\begin{array}{l}\text { A list of values or a mathematical function that defines the relative amounts of particles present, sorted according to size - } \\
\text { modality, peak magnitude, minimum, and maximum are included }\end{array}$ \\
A geometric description of the extremities of a nanomaterial (eg, I-D, 2-D, 3-D) along with the rigidity of its spatial \\
arrangement, as represented in its external surface
\end{tabular}

Notes: Those PCCs marked with an asterisk were described based on the International Organization for Standardization's (ISO) descriptions. Divergence from any standard descriptions occurred based on the scope of data curated into the Registry. 
MIAN may be modified to reflect new knowledge, including new instruments, new or modified protocols, and new best practices.

\section{Instance of characterization}

Any nanomaterial characterization, whether for PCC or for interaction with biological or environmental systems comes with essential information regarding the nanomaterial's immediate history, such as its synthesis or its most recent processing history. The metadata surrounding this immediate history have been classified into three categories of IOC $^{5,18}$ to track a nanomaterial's synthesis or process history and to validate or invalidate comparisons of data when the user is evaluating multiple nanomaterial entries. The three categories of IOC are:

- As synthesized - native state of the nanomaterial when synthesized

- As received - native state of the nanomaterial when purchased or received

- As processed - nonnative state of the nanomaterial modified since synthesized or received.

With each category, particular metadata are curated about the specific circumstances. Table 2 presents the fields of metadata designed in the minimal information standard to fully describe the IOCs and to provide adequate details for precise nanomaterial comparison and analysis.

\section{Compliance level}

In order to improve the overall quality of information about nanomaterials in diverse studies, the compliance-level feature of the Registry provides a metric on the quality and quantity of characterization for each nanomaterial entry. In the many cases where appropriate standards for nanomaterial characterization do not yet exist, the compliance-level score serves

Table 2 Minimal information curate for each instance of characterization (IOC) category

\begin{tabular}{ll}
\hline $\begin{array}{l}\text { Instance of } \\
\text { characterization }\end{array}$ & Minimal information curated \\
\hline As synthesized & - Manufacturer or laboratory name \\
& - Product name \\
- Lot number & - General synthesis description \\
- DOl citation of synthetic procedure & - Manufacturer or laboratory name \\
- Product name & - Lot number \\
As received & - Processing details (ex aerosolized, \\
& suspended, dried, milled, heated, etc) \\
As processed &
\end{tabular}

to identify the data gaps in which more research emphasis may be needed. Figure 1 shows the different compliance levels assigned to particular ranges of compliance scores, with gold being $76 \%-100 \%$, silver being $51 \%-75 \%$, bronze being 26\%-50\%, and merit being 0\%-25\%. Calculation of compliance levels for each individual PCC $\left(\mathrm{CL}_{\mathrm{IPCC}}\right)$ is made in cases where a nanomaterial was characterized (1), and each nanomaterial entry in the Registry is given a compliance level for its overall PCC characterization $\left(\mathrm{CL}_{\mathrm{OPCC}}\right)(2)$,

$$
\begin{gathered}
\mathrm{CL}_{\mathrm{IPCC}}=\sum_{\mathrm{i}=1}^{\mathrm{M}} \mathrm{W}_{\mathrm{i}} /(\mathrm{M} * \mathrm{n}) \\
\mathrm{CL}_{\mathrm{OPCC}}=\sum\left(\mathrm{CL}_{\mathrm{IPCC}} * \mathrm{~W}_{\mathrm{IPCC}}\right) / \mathrm{n}
\end{gathered}
$$

where $\mathrm{i}=$ measurement number, $\mathrm{M}=$ number of measurements, $\mathrm{n}=$ total number of points possible, and $\mathrm{W}=$ the assigned weighting factor. $\mathrm{ACL}_{\mathrm{IPCC}}$ is reached, first, through a comparison to the MIAN. When a nanomaterial entry is curated from a data source, all the data are curated to the streamlined vocabulary of the MIAN, and all data fields and types have assigned weighting factors. Each piece of curated data receives the applicable weighting factor and is incorporated into the $\mathrm{CL}_{\mathrm{IPCC}}$ summation. In general, no part of the data or metadata is weighted more heavily, so reporting more thoroughly in areas of experimental protocol, instrumentation, and best-practice metadata can quickly increase a PCC's compliance by two levels. The example in Table 3 gives three levels at which a characterization for size may be reported in the literature. While characterization 1 only reported

\section{Compliance levels}
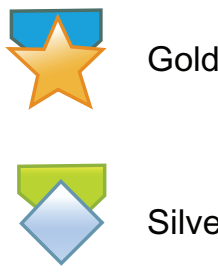

Silver

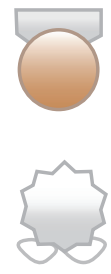

Bronze

Merit

Figure I Nanomaterial Registry compliance medals in decreasing order of compliance score range: gold, silver, bronze, and merit. 
Table 3 Three examples of reported data from a size characterization along with the respective calculated compliance scores and levels

\begin{tabular}{|c|c|c|c|}
\hline Size characterizations & $\mathbf{I}$ & 2 & 3 \\
\hline Curated data & $\begin{array}{l}\text { Mean } \\
\text { Diameter }=65 \mathrm{~nm}\end{array}$ & $\begin{array}{l}\text { Mean hydrodynamic } \\
\text { diameter }=65 \mathrm{~nm} \\
\text { by Dynamic Light } \\
\text { Scattering }\end{array}$ & $\begin{array}{l}\text { Mean hydrodynamic diameter, } \\
\text { intensity weighted }=65 \mathrm{~nm} \pm 4 \mathrm{~nm} \\
\text { by Dynamic Light Scattering } \\
\text { Protocol: ASTM E2490-09 } \\
\text { Instrument: } \\
\text { Malvern ZetaSizer nano ZS } \\
\checkmark \mathrm{pH} \text { of suspension }=8 . \mathrm{I} \\
\checkmark \text { temperature of suspension }=25^{\circ} \mathrm{C} \\
\checkmark \text { solvent/medium type }=\text { phosphate buffered saline }\end{array}$ \\
\hline Compliance score & $25 / 91=27.47 \%$ & $5 I / 91=56.04 \%$ & $74 / 91=81.32 \%$ \\
\hline Compliance level & Bronze $\bar{\varnothing}$ & Silver $\nabla$ & Gold \\
\hline
\end{tabular}

Notes: The difference for a size characterization between a bronze level and silver level could be in the reporting of the technique used, while to reach a gold level, more details on the instrumentation and protocol would be needed.

Abbreviation: ASTM, American Society for Testing and Materials.

the mean diameter of the nanoparticle, characterization 2 reported the technique used to perform the characterization, and thus received a higher compliance level. Characterization 3 reported not just the data on size but also the error for the measurement, the computational method by which the measurement was calculated (intensity-weighted), and the characterization protocol and parameters. This resulted in a gold compliance level for characterization 3.

For the calculation of the $\mathrm{CL}_{\mathrm{OPCC}}$, initial weighting factors have been assigned to each PCC in order to stress a hierarchy of importance for PCC characterization. The default Registry MIAN PCC weighting factors place composition, size, and surface chemistry as some of the highest-weighted PCCs. Aggregation/agglomeration state and purity are among the lowest-weighted PCCs. Over time, these weighting factors could be modified as new information becomes available. Also, understanding that each stakeholder group potentially identifies with different hierarchies of PCC, customized stakeholder weighting factors will be incorporated into the Registry in the future. Like the MIAN, updating the calculation of compliance levels based upon new information as it comes available is an inherent feature of the Registry.

\section{Features of the Nanomaterial Registry web-based tool}

The data in the Registry are available via browse as well as basic and advanced searches. The basic search allows the user to not only search by keyword but also to find all entries in which certain PCCs have been characterized. The advanced search offers extensive power to find exact data values of PCC characterization. Unique features of the Registry tool center on data analysis and include the compliance levels, side-byside comparison tool, and nanomaterial-matching tool.
The search and browse results page was designed in tabular format in order to show the user not just what PCCs were characterized for each nanomaterial but also the compliance levels for the PCC characterization. All nanomaterial entries resulting from a search or browse query are listed in the table with their overall compliance level, individual PCC compliance levels, and indications of whether the nanomaterial entry was studied in a biological or environmental system. From any results table, the user then has the ability to compare nanomaterials side by side by selecting up to three entries of interest. Any individual nanomaterial entry can be viewed by clicking on its Registry numeric identifier. Preliminary human-readable descriptions are also available for each nanomaterial entry and are a very brief summary of the nanomaterial's chemical makeup and shape. As standards for human-readable names are developed, the Registry will be updated to adopt those standards.

Side-by-side comparison of nanomaterial characterizations may be used via any results table. Once the user chooses up to three nanomaterial entries to compare, they then select the individual data sets of IOC in which they are interested. The comparison tool then displays all available PCC characterization data side by side, and the IOCs chosen for comparison may be easily changed.

A simple, rule-based matching system has been established for data in the Registry. The matching tool can be reached from any nanomaterial entry's individual page via the "Find similar nanomaterials" button. This query produces all nanomaterial entries that are at least $10 \%$ similar to the original nanomaterial entry. Percentages of similarity are awarded based on the nanomaterial entries' IOCs, sizes, shapes, surface chemistries, and isoelectric points. The rules for matching will evolve as the complexity of available data in the Registry evolves. 


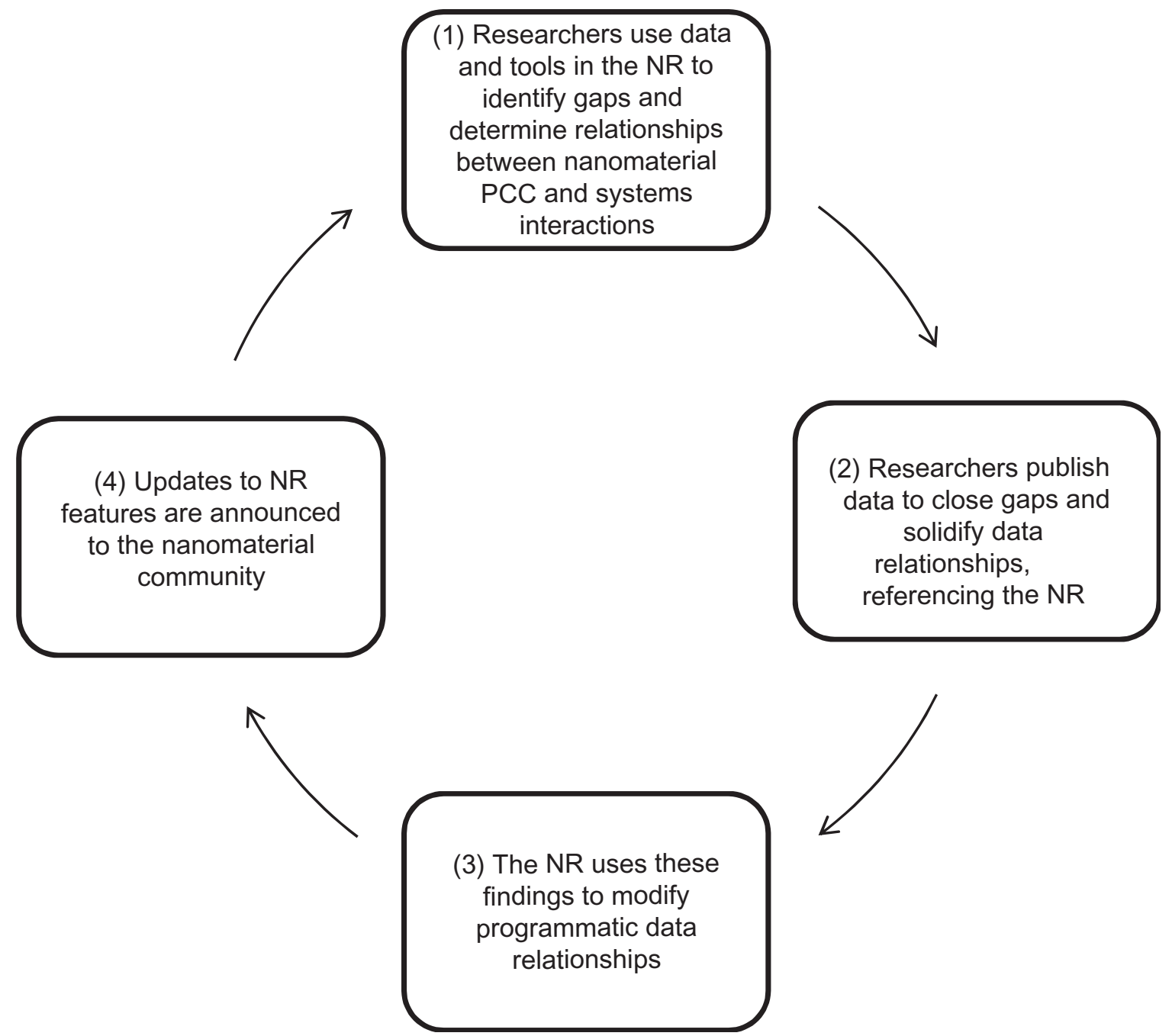

Figure 2 Process by which growth is expected for the Nanomaterial Registry (NR). Abbreviation: PCC, physicochemical characterization.

\section{Conclusion}

The data currently available in the Registry were curated from already-existing public databases and resources: caNanoLab, ${ }^{19}$ InterNano, ${ }^{20}$ and the Nanoparticle Information Library (NIL). ${ }^{21}$ In the future, the Registry will include curated data from additional data sources.

A beta version of the Nanomaterial Registry, available at http://www.nanomaterialregistry.org, was released to the public in June 2012. Containing 255 nanomaterial entries, the beta version offered the following tool features for curated PCC data: browse, search, compliance ratings, side-by-side comparison, and matching. The data model and website features for studies on nanomaterial interactions with biological and environmental systems were completed soon thereafter, and curated study data will be available to the public in the near future with a full release of the Registry site. Also, the
Registry recognizes the importance of using ontologies and controlled vocabularies and will continue to incorporate standards into the programmatic practices of the Registry as they become available.

The Registry was developed to be a useful tool that will grow organically based on a cyclical relationship with the nanomaterial community (Figure 2). Over time, the Registry will have tremendous potential for significant long-term impacts, including:

- aiding in the development of new models, assays, standards, and manufacturing methods

- accelerating the translation of new nanomaterials for biomedical and environmental applications

- promoting standards in nanomaterial characterization testing, handling, and disposal, and

- supporting science-based regulatory decision-making. 


\section{Acknowledgments}

This project has been funded by the National Institute of Biomedical Imaging and Bioengineering (NIBIB) and the following partners: the National Institute of Environmental and Health Sciences (NIEHS), and the National Cancer Institute (NCI), National Institutes of Health, Department of Health and Human Services, under contract no HHSN268201000022C.

\section{Disclosure}

The authors report no conflicts of interest in this work.

\section{References}

1. Wijnhoven SWP, Peijnenburg WJGM, Herberts CA, Hagens WI. Nano-silver - a review of available data and knowledge gaps in human and environmental risk assessment. Nanotoxicology. 2009;3(2):109-138.

2. Hackley VA, Fritts M, Kelly JF, Patri AK, Rawle AF. Enabling standards for nanomaterial characterization. InfoSIM. Aug 2009:24-29.

3. Hull M, Bowman D. Nanotechnology Environmental Health and Safety: Risks, Regulation and Management [e-book]. Oxford, UK: Elsevier; 2009.

4. Warheit DB. How meaningful are the results of toxicological studies in the absence of adequate material characterization? Toxicol Sci. 2008; 101(2):183-185.

5. Sayes CM, Warheit DB. Characterization of nanomaterials for toxicity assessment. Wiley Interdiscip Rev Nanomed Nanobiotechnol. 2009;1(6): 660-670.

6. Maynard AD, Aitken RJ, Butz T, et al. Safe handling of nanotechnology. Nature. 2006;444(7117):267-269.

7. Hussain SM, Braydich-Stolle LM, Schrand AM, et al. Toxicity evaluation for safe use of nanomaterials: recent acheivements and technical challenges. Adv Mater. 2009;21(16):1549-1559.

8. Boverhof D, David R. Nanomaterial characterization: considerations and needs for hazard assessment and safety evaluation. Anal Bioanal Chem. 2010;396(3):953-961.

9. Characterization Matters [webpage on the Internet]. The Parameters List. Ann Arbor (MI): Minimum Information for Nanomaterial Characterization Initiative; 2009. Available from: http://characterizationmatters.org/ parameters. Accessed October 10, 2012.
10. Organisation for Economic Co-operation and Development Guidance Manual For the Testing of Manufactured Nanomaterials: OECD's Sponsorship Programme; First Revision. Paris: OECD; 2010. Available from: http://search.oecd.org/officialdocuments/ displaydocumentpdf/? cote $=$ env $/ \mathrm{jm} /$ mono $\% 282009 \% 2920 /$ rev\&doclanguage $=$ en. Accessed October 10, 2012.

11. ASTM International. ASTM WK28974: New specification for a standard file format for the submission and exchange of data on nanomaterials and characterizations. 2010. Available from: http://www.astm. org/DATABASE.CART/WORKITEMS/WK28974.htm. Accessed February 26, 2013.

12. International Organization for Standardization. ISO/TS 27687:2008. Nanotechnologies: Terminology and Definitions for Nano-Objects - Nanoparticle, Nanofibre and Nanoplate. Geneva: ISO; 2008.

13. Thomas DG, Pappu RV, Baker NA. Nanoparticle ontology for cancer nanotechnology research. J Biomed Inform. 2011;44(1):59-74.

14. Ostraat MO, Mills KC, Guzan KA. The Nanomaterial Registry: opportunities and challenges in informatics. Paper presented at the IEEE International Conference on Bioinformatics and Biomedicine; October 4-7, 2012; Philadelphia, USA.

15. Characterization Matters [homepage on the Internet]. Available from: http://characterizationmatters.org. Accessed October 10, 2012.

16. International Organization for Standardization. ISO/TS 80004-4: 2011. Nanotechnologies: Vocabulary - Part 4: Nanostructured Materials. Geneva: ISO; 2011.

17. International Organization for Standardization. ISO/TR 13014:2012. Nanotechnologies: Guidance on Physico-Chemical Characterization of Engineered Nanoscale Materials for Toxicologic Assessment. Geneva: ISO; 2012.

18. Sayes C, Berg JM. Physical and chemical properties of nanomaterials relevant to toxicological studies, in vitro and in vivo. In: Sitharaman B, editor. Nanobiomaterials Handbook [e-book]. Boca Raton (FL): CRC Press; 2011:1-14.

19. Cancer Nanotechnology Laboratory [homepage on the Internet] Available from: https://cananolab.nci.nih.gov/caNanoLab. Accessed February 26, 2013

20. InterNano [homepage on the Internet]. Available from: http://www. internano.org. Accessed February 26, 2013.

21. Nanoparticle Information Library (NIL) [homepage on the Internet] Available from: http://www.nanoparticlelibrary.net. Accessed February 26, 2013
International Journal of Nanomedicine

\section{Publish your work in this journal}

The International Journal of Nanomedicine is an international, peerreviewed journal focusing on the application of nanotechnology in diagnostics, therapeutics, and drug delivery systems throughou the biomedical field. This journal is indexed on PubMed Central, MedLine, CAS, SciSearch ${ }^{\circledR}$, Current Contents $₫ /$ Clinical Medicine,

\section{Dovepress}

Journal Citation Reports/Science Edition, EMBase, Scopus and the Elsevier Bibliographic databases. The manuscript management system is completely online and includes a very quick and fair peer-review system, which is all easy to use. Visit http://www.dovepress.com/ testimonials.php to read real quotes from published authors. 